Horizons philosophiques

\title{
La science n'est pas toujours neutre : biodiversité, commercialisation et génétique des populations
}

\section{Pierre Gendron}

Volume 7, numéro 1, automne 1996

Le dernier pont

URI : https://id.erudit.org/iderudit/801031ar

DOI : https://doi.org/10.7202/801031ar

Aller au sommaire du numéro

Éditeur(s)

Collège Édouard-Montpetit

ISSN

1181-9227 (imprimé)

1920-2954 (numérique)

Découvrir la revue

Citer cet article

Gendron, P. (1996). La science n'est pas toujours neutre : biodiversité, commercialisation et génétique des populations. Horizons philosophiques, 7(1), 85-96. https://doi.org/10.7202/801031ar d'utilisation que vous pouvez consulter en ligne. 


\section{LA SCIENCE N'EST PAS TOUJOURS NEUTRE : BIODIVERSITÉ, COMMERCIALISATION ET GÉNÉTIQUE DES POPULATIONS*}

Le patrimoine génétique humain doit-ilêtre considéré comme une "ressource" (au même titre, par exemple, que le génome des plantes)? Cette question à première vue étonnante a surgi récemment dans le débat entourant l'examen de certains aspects d'un programme mondial de recherche en génétique des populations connu sous le nom de «Human Genome Diversity Project» (HGDP). Ce débat concerne à la fois les puissants moyens techniques qu'a réussi à mobiliser la vaste entreprise en cours de séquençage du génome humain, sous l'égide du "Human Genome Organisation" (HUGO), et les instruments juridiques internationaux qui ont pour objet la préservation de la biodiversité animale et végétale.

Aujourd'hui, en effet, il est permis de se demander si certaines des visées "utilitaristes" " inscrites dans le processus qui a abouti à la Conférence de Rio en 1992, et qui est à l'origine

Le texte du présent article est le résultat d'un séminaire donné à l'Université de Montréal, le 4 avril 1996. Depuis, une nouvelle donnée est venue s'ajouter à la problématique de la biodiversité telle qu'elle était alors connue de l'auteur. L'exposé n'a pu tenir compte du fait que les pays représentés à la deuxième Conférence des Parties à la Convention sur la diversité biologique, qui a eu lieu à Djakarta en novembre 1995, ont convenu que les ressources génétiques humaines ne relevaient pas de la Convention sur la diversité biologique. L'auteur remercie Thérèse Leroux, du Centre de recherche en droit public, pour lui avoir communiqué cette information. Merci également à Marie-Hélène Parizeau, de la Chaire de recherche en éthique de l'environnement de l'Université McGill, pour son aide (et particulièrement pour la consultation de son exemplaire du Guide to the Convention on Biological Diversity).

1. L'emploi des termes "utilitaire" et "utilitariste» ne fait ici que suivre l'usage courant, sans référence particulière à la théorie connue en éthique sous le nom d'utilitarisme. Le point de vue adopté esten faitceluide Marshall Sahlins, professeur d'anthropologie à l'Université de Chicago, qui explique dans un ouvrage intitulé $A u$ cœur des sociétés : Raison utilitaire et raison culturelle, (traduit de l'anglais par Sylvie Fainzang, Paris, Gallimard, 1980) : “L'utilité peut être considérée dans des 
de la Convention sur la diversité biologique, ne s'étendront pas bientôt au génome humain. Dans la mesure où certaines populations humaines peuvent être considérées comme les dépositaires de ressources génétiques, rien ne semble a priori pouvoir empêcher une telle évolution de se produire. Rien ne permet non plus d'affirmer, d'un point de vue scientifique ou autrement, que cette évolution soit inévitable et qu'elle doive se produire. Toutefois, du moment qu'elle est possible, il serait imprudent de ne pas s'interroger et de ne pas chercher à en déterminer les conditions, ni d'essayer d'en prévoir les conséquences sociales, éthiques et juridiques ${ }^{2}$.

dimensions subjectives et objectives, bien que de nombreuses théories négligent de spécifier la logique pratique qu'elles prennent comme base de l'ordre culturel. Pour certaines, cependant, il est clair que la culture est un précipité de l'activité rationnelle des individus agissant en fonction de leurs intérêts. Ceci est de l' "utilitarisme" dans toute l'acception du mot; sa logique est la maximisation des rapports entre moyens et fins. Les théories de l'utilité objective sont naturalistes ou écologiques. Pour elles, la sagesse matérielle substantialisée en forme culturelle est la survie de la population humaine ou d'un ordre social donné. La logique précise est celle de l'avantage adaptatif, ou du maintien du système dans des limites naturelles de viabilité. Par opposition (...), (ce livre) pose comme qualité distinctive de l'homme non pas le fait qu'il doit vivre dans un monde matériel, condition qu'il partage avec tous les organismes, mais le fait qu'il vit conformément à un schème signifiant qu'il a lui-même forgé, ce en quoi il est unique" (p. 7-8).

2. La question d'une "éthique des priorités" dans l'allocation des budgets de recherche garde cependant toute sa pertinence, commel'indique entre autres le témoignage de Carl Seguin, chercheur en biologie moléculaire à l'Université Laval, lors d'un colloque tenu dans le cadre du Congrès de l'ACFAS, à l'Université de Sherbrooke, le 22 mai 1991 :

Je crois plus profitable de favoriser les démarches fondamentales des différents laboratoires dans le monde que de payer pour des "gros monstres" avec de belles machines de séquençage qui vont favoriser le développement de l'industrie mercantile et capitaliste qui désire séquencer l'ensemble du génome humain sous prétexte, dit-elle, qu'on va probablement sauver des vies humaines, qu'on va probablement améliorer le pronostic de je ne sais quelles maladies humaines. Cela m'apparaît démagogique, irrationnel et dépourvu d'imagination" (cf. Le génome humain: une responsabilité scientifique et sociale, Sainte-Foy, Presses de l'Université Laval, 1992, p. 150).

Et comme l'explique Susan Lindee, du Département d'histoire et de sociologie des sciences de l'Université de Pennsylvanie :

"The National Institutes of Health (NIH) and the Department of Energy - the primary funding agencies for genome mapping in the United States - have each set aside 5 percent of their genome budgets to supportscholars studying the ethical, legal, and social implications (commonly abreviated as ELSI) of the genome project. (...) Certainly it is a quality-control division that has already manifested some independence of mind, as those who are funded by the $\mathrm{NIH}$ or the Department of 
Qu'est-ce que la biodiversité? La diversité est une caractéristique de la vie comme telle et s'exprime à trois niveaux : les variétés (diversité génétique ou intraspécifique), les espèces (diversité interspécifique) et les écosystèmes ${ }^{3}$. L'espèce humaine constitue un cas à part, il convient de le noter, car il n'y a qu'une espèce dite humaine et celle-ci a pour habitat la terre entière (l'écosystème planétaire). II n'en demeure pas moins vrai que, pour l'ensemble du monde vivant, chaque écosystème est formé par l'association d'une communauté vivante et d'un environnement physico-chimique spécifique. Et c'est pourquoi la préservation de la diversité biologique ne concerne pas seulement les espèces, mais aussi les espaces ou habitats ${ }^{4}$.

L'interdépendance du monde vivant est donc au fondement de toute définition de la biodiversité. C'est la première chose qu'il faut prendre en considération lorsqu'il s'agit d'évaluer l'importance de la biodiversité. Cela dit, l'évaluation économique de la biodiversité dépend surtout d'un certain nombre de facteurs culturels : considérations utilitaristes (les changements

Energy seem perfectly willing to interpret the (Human Genome Project) as a social menace. ELSI funding has not turnet its very diverse grantees into uniform champions of mapping and sequencing genes.

"What the funding has done, however, is to promote the distressing general tendency to divide the project into "science" and the "implications" of that science. Officially outside the ELSI territory is the most important question of all : Is mapping the human genome a meaningful scientific priority?" (cf. The ELSI Hypothesis, Isis, 85 (1994), p. 294 et 296).

3. En dehors des trois niveaux classiques, il existe encore d'autres expressions de la biodiversité : “l'abondance relative des espèces, la structure d'âge des populations, la distribution des populations dans une région, les changements dans le temps de la composition des communautés, et aussil'ensemble des processus de prédation, de parasitisme et de symbiose" (cf. Daniel Chevallier, Rapport sur la biodiversité et la préservation du patrimoine génétique, Tome 1 (Conclusions du rapporteur), Paris, Office parlementaire d'évaluation des choix scientifiques et technologiques, 1992, p. 13).

4. "The loss of individuals and populations through, among other things, habitat destruction narrows the gene pool of a species and restricts its adaptational or evolutionary options. Therefore, if maintained, genetic diversity increases the chances for a species' survival. (...) Humans have relied on genetic diversity to create a wide range of genetically diverse crops, animals and micro-organisms which have enhance their own survival» (Lyse Glowka, Françoise BurhenneGuilmin et Hugh Synge, $A$ Guide to the convention on Biological Diversity, Cambridge (UK), IUCN, 1994, p. 21). 
défavorables à l'environnement, la biodiversité comme ressource pour l'agriculture, la biotechnologie et l'industrie pharmaceutique); aspects esthétiques (le spectacle de la nature en tant qu'il est, pour plusieurs, au fondement de la culture); fondements éthiques, enfin (la protection du mode de vie traditionnel des populations autochtones, la valeur de préservation pour les générations futures, le «droit d'exister» des espèces sauvages, indépendamment des bénéfices qu'elles peuvent fournir à l'humanité). Les économistes ont été sollicités pour mesurer l'utilité et la valeur de la diversité biologique; mais leur évaluation comporte de nombreuses incertitudes et ne peut prétendre à elle seule orienter les stratégies de préservation. La protection de la nature impose de prendre en compte les dimensions culturelles, historiques et géopolitiques de la diversité biologique, ce qui ne peut se faire sans une véritable concertation à l'échelle internationale ${ }^{5}$.

Parmi les instruments juridiques d'une telle concertation, il faut maintenant compter la Convention sur la diversité biologique issue de la Conférence des Nations Unies sur l'Environnement et le Développement qui a eu lieu en juin 1992 à Rio de Janeiro, et dont les objectifs sont : a) la conservation de la biodiversité (considérée dans son ensemble); b) l'utilisation durable de ses éléments; et $c$ ) le partage équitable des bénéfices provenant de l'utilisation des ressources génétiques ${ }^{6}$. Une perspective s'ouvre donc sur la commercialisation; car, à la fin, l'accent n'est plus mis surl'environnement mais plutôt surle développement ${ }^{8}$.

5. Cf. François Lévêque et Matthieu Glachant, "Diversité génétique : La gestion mondiale des ressources vivantes", La Recherche, 23 (1992) p. 114-115.

6. L'emploi du terme "génétique annonce déjà, dans ce premier article, le thème de la (bio)technologie, qui est omniprésent dans la Convention (et pour cause) : "Increased progress in biotechnology has provoked greater attention to the value of genetic resources - well beyond the narrow scope of new plant varieties" (ibid., p. 5).

Ce phénomène est renforcé, du reste, par l'équation communément faite entre génie génétique et biotechnologie. Comme le font remarquer Glowka et al. dans leur commentaire : "To many people genetic engineering "is" biotechnology. With genetic engineering techniques, a gene for a particular trait from one organism can be directly inserted into another, even if the two organisms are not from the same spectes. This is a major advance over conventional plant and animal breeding where traits can only be imparted to organisms indirectly and then usually between organisms of the same species" (ibid., p. 21). 
L'éventualité d'une application de la Convention à la diversité génétique humaine peut aussitôt être envisagée : il suffit de considérer certains éléments ou produits du génome humain comme autant de «ressources». Un prototype d'action, du reste, se trouve peut-être dans l'exemple d'un petit pays d'Amérique centrale, le Costa Rica, qui possède de très importantes réserves naturelles sur son territoire.

Pour inventorier la biodiversité du Costa Rica, qui est estimée à quelque 500000 espèces, un institutà but non lucratif a été créé sous le nom de «Instituto de Biodiversidad» (INBIO). Cet institut a entrepris une collecte systématique d'échantillons représentatifs de la biodiversité du pays (cette collecte est effectuée par des «parataxinomistes», paysans ayant reçu une formation taxinomique de base). Les échantillons sont ensuite étudiés et classés par des techniciens et des systématiciens compétents. Ce travail a pu se prolonger et s'amplifier en vertu d'un accord passé avec la société pharmaceutique Merck. Le contrat prévoyait que Merck pouvait évaluer l'intérêt pharmaceutique ou agrochimique de certains végétaux, insectes et micro-organismes présents au Costa Rica. Si des produits étaient mis sur le marché à partir des molécules ainsi évaluées, des redevances devaient être payées à I'INBIO. L'élément essentiel à retenir de cette expérience est que les dirigeants de I'INBIO se soient convaincus que «la meilleure façon de préserver la biodiversité était de montrer qu'en la protégeant, en l'étudiant et en l'exploitant intelligemment et précautionneusement, il était possible de créer une source de revenu régulière pour les habitants du pays ${ }^{8 n}$.

7. Un tel glissement pourrait sans doute être qualifié de dérive "anthropocentrique». Mais l'approche biocentrique a aussi ses inconvénients, comme le fait remarquer André Beauchamp, ex-président du BAPE (Bureau d'audiences publiques sur l'environnement) :

L'adoption d'un point de départ carrément biocentrique postulant la négation d'une démarche anthropocentrique me paraît dangereuse bien qu'à première vue elle se présente sous des dehors généreux. Son application stricte exigerait en réalité l'établissement d'un système autoritaire et l'exclusion des pauvres. Elle signifierait aussila mainmise d'une éthique biologique sur les conduites humaines, escamontant la spécificité de l'espèce humaine : la dimension culturelle. (Introduction à l'éthique de l'environnement, Montréal, Éditions Paulines, 1993, p. 89).

8. Cf. Daniel Chevallier, op. cit., p. 98-99. 
Or, la question qui se pose maintenant est celle de savoir s'il y a lieu d'inclure parmi les ressources visées par ce type de contrat, et plus généralement par les efforts de préservation de la biodiversité, le patrimoine génétique humain (ou du moins certaines populations humaines). II n'y a pas si longtemps, les propos tenus par Walter Bodmer et Luigi Cavalli-Sforza, impliqués dans la mise sur pied du HGDP (dont il a été question plus haut), ont pu le laisser croire. Selon eux, les puissants outils de la génétique moléculaire auraient permis de réaliser une étude systématique de diverses populations, choisies pour représenter l'ensemble de l'humanité, avec constitution de banques de données informatisées sur leur patrimoine génétique. Cette étude aurait d'abord compris l'échantillonnage des populations en cause (prises de sang, etc.) et ensuite, point capital, la transformation des échantillons ainsi recueillis en «lignées cellulaires», pour fins de conservation et d'utilisation ultérieure, notamment dans l'analyse du polymorphisme humain?.

L'intérêt scientifique du HGDP tiendrait d'abord au fait que la seule définition des populations humaines qui soit vraiment rigoureuse d'un point de vue scientifique est celle qui se fonde sur la distribution des fréquences géniques. L'étude de cette distribution constituerait la base des analyses qui permettraient d'établir, d'après Cavalli-Sforza, certaines relations entre les populations, telles que les généalogies, la configuration des phénomènes migratoires et le parallélisme entre diversité génétique et diversité linguistique ${ }^{10}$. En outre, l'importance pratique du projet viendrait des possibilités nouvelles qu'il offrirait à l'épidémiologie génétique. En effet, comme le rappelle Bodmer, les enquêtes épidémiologiques qui associent certains marqueurs génétiques à certaines maladies ont besoin du point de comparaison que constituent les données relatives à la

9. Walter Bodmer, The Case for the Human Genome Diversity Project. Londres, Human Genome Organisation, 1993, p. 4.

10. Ibid., p. 6. 
distribution des marqueurs génétiques dans des populations dites normales. Et c'est ce genre de contribution qu'apporterait le HGDP ${ }^{11}$.

La réalisation d'un tel projet ne serait pas sans poser certains problèmes du point de vue d'une éthique de type classique : consentement, conformité aux normes relatives à la protection des sujets humains, confidentialité, respect des cultures et des traditions orales, risques de conflits d'intérêt et risques de mauvaises utilisations des résultats et d'interprétations favorables à l'eugénisme ou au racisme. Mais la chose qui semble susciter à la fois les plus grands espoirs et les plus vives inquiétudes, c'est la perspective d'une commercialisation possible de "produits du corps humain" (sous la forme des lignées cellulaires obtenues, le cas échéant, à partir de recherches en génétique des populations effectuées sur le terrain). Malgré toutes les prises de position contre la commercialisation du projet sur la diversité génétique humaine en tant que tel, c'est-à-dire comme projet de recherche scientifique, un rapprochement n'a pas manqué d'être fait avec d'autres situations où l'appropriation des ressources génétiques pouvait impliquer l'existence de brevets ou de contrats. La rémunération, les droits de propriété intellectuelle et les transferts de technologie pourraient donc à l'avenir faire l'objet de revendications à la lumière de la Convention sur la diversité biologique $^{12}$.

La situation risque de devenir problématique, en somme, dans la mesure où les échantillons recueillis par les équipes du HGDP seraient effectivement destinés à subir une étape de

11. Ibid., p. 8.

12. "Many (developing countries) made their participation in the negotiations conditional on the inclusion in the Convention of obligations and measures on three types of access : access to genetic resources, which they wished to have recognized as subject to national authority; access to relevant technology, stressing that it includes biotechnology; and access for the providing States to benefits ultimately gained from the use of genetic material in the development of biotechnology. This evolution is (...) grounded in practical reasoning : control over access to genetic resources gives the providing Party the opportunity to negotiate the mutually agreed terms for fair and equitable sharing of benefits required by article 15" (Glowka et al., op. cit., p. 5). 
transformation (lignées cellulaires humaines). Or, dans le cas présent, qui dit transformation ditéventuellement biotechnologie, voire industrie. D'où certains doutes et certaines craintes; d'où, également, le débat éthique entourant la commercialisation.

"Le monde occidental, comme le dit la juriste Marie-Angèle Hermitte, du CNRS (Groupe d'analyse des politiques publiques), est passé relativement rapidement d'une situation où l'idée de commercialisation du corps humain évoquaitl'esclavage, à une situation où elle est liée à de fabuleux progrès médicaux et pharmacologiques : sang, organes, matériel génétique, tout peut être utilisé à des fins thérapeutiques ou scientifiques, voire purement commerciales, faisant du corps humain une "source de matières premières" pour l'industrie." D'où l'obligation de "procéder avec la plus grande prudence pour éviter le retour à des formes d'exploitation qui, pour être moins violentes que l'esclavage, n'en seraient pas moins attentatoires à la dignité humaine ${ }^{13^{\prime}}$.

Par conséquent, la controverse qui entoure le projet sur la diversité génétique humaine risque de s'intensifier à mesure que seront progressivement dévoilés ses véritables enjeux. La nécessité d'un examen sérieux de certaines conséquences logiques de l'intégration du patrimone génétique humain dans la problématique générale de la biodiversité est évidente; mais il n'est pas moins clair, en raison de la normativité sous-jacente aux choix scientifiques et technologiques, que cet examen devra aussi englober certaines questions de politique scientifique et de financement de la recherche. Et c'est pourquoi il importe de bien saisir l'arrière-plan historique des travaux préparatoires à la Conférence de Rio.

Comme le rappellent Michel Chauvet et Jean-Paul Galland : "Le climat de la négociation s'est très vite dégradé entre pays du Nord et pays du Sud sur les questions d'accès aux ressources de la bioéthique. Bruxelles, De Boek-Wesmael, 1993, p. 76. 
génétiques et aux biotechnologies, présentées par certains pays en développement comme "les deux revers d'une même médaille». Ce "couplage" s'insère dans le problème plus large des relations entre environnement et développement. D'une manière caricaturale, l'argumentation se résume par la revendication d'une égalité des échanges entre pays du Sud, riches en gènes, et pays du Nord, riches en technologies ${ }^{14}$ ". Le plus grave, selon eux, c'est l'approche utilitaire qui a prévalu dans le débat sur l'accès aux biotechnologies etle «partage des bénéfices». Ainsi qu'ils le constatent : «La biotechnologie est maintenant perçue comme le moyen pour les pays en développement d'exploiter à des fins économiques l'énorme potentiel que leur offre la diversité biologique ${ }^{15}$ ».

Deux visions s'affrontent sur la question de l'environnement. Pour les uns, l'être humain doit être considéré comme un des éléments de la Nature; pour les autres un bon environnement se modèle sur les besoins des hommes. Sans vouloir trancher ce débat, une étude comparée devrait permettre de tirer des leçons de certaines expériences dans le domaine de la biodiversité et de les appliquer au cas du patrimoine génétique humain, notamment en ce qui concerne l'élaboration des normes et le rôle que peut y jouer la «technique juridique».

La première organisation juridique de l'accès auxorganismes vivants a pris la forme d'un Engagement (Undertaking), élaboré en 1983 sous l'égide de la FAO (Food and Agriculture Organization), qui posa le principe selon lequel les végétaux, les «ressources phytogénétiques», étaient «patrimoine commun de l'humanité», en libre accès à tous. Or, les pays du Sud ont par la suite mis en cause le pouvoir de prélever sur leur territoire

14. Michel Chauvet et Jean-Paul Galland, "La diversité biologique et les ressources génétiques", Droit et génie génétique, Paris, Elsevier, 1994, p. 26.

15. Ibid., p. 28.

Cf. aussi André Beauchamp, op. cit., p. 95 : “Le Sommet de la Terre, tenu à Rio de Janeiro en 1992, a marqué une certaine tension, sinon un échec, entre une problématique du milieu naturel mise de l'avant par les pays riches et une problématique du besoin des pauvres issue des pays du Sud". 
des organismes vivants pour ensuite les modifier génétiquement et, dans certains cas, les renvoyer brevetés dans leur pays d'origine. Ils ont finalement mis de côté le concept de patrimoine commun de l'humanité et on tabouti en 1989 à une interprétation plus restrictive de l'Engagement de la FAO, comme le souligne Marie-Angèle Hermitte : «Si les ressources phytogénétiques restèrent patrimoine commun de l'humanité, on récusa le libre accès gratuit. II fallait désormais demander une autorisation avant de faire une collecte; si l'on prenait un organisme vivant et qu'on le transformait pour le mettre dans le circuitéconomique, on admit qu'il faudrait payer quelque chose au pays d'origine, sur le fondement de ce que l'on a appelé le "droit des agriculteurs» ${ }^{16}$ ». Pour la juriste du CNRS, "la Convention de Rio n'est qu'une sorte d'extension du système du droit des agriculteurs mis en place par la FAO", puisque désormais tout accès à un fragment d'ADN d'un organisme vivant, "qu'il soit végétal ou animal (et pourquoi pas humain, puisque l'être humain n'est pas expressément exclu du champ d'application de la Convention)", doit donner lieu à autorisation et rémunération ${ }^{17}$.

Mais ce n'est pas tout. Une deuxième transformation dans l'accès aux organismes vivants dans les pays du Sud concerne l'humanité, puisque les biotechnologies permettent d'utiliser le corps humain lui-même comme ressource. L'expérience des banques de sang, organisée juridiquement après la deuxième guerre mondiale, a conduit à penser qu'il fallait créer une sorte de troisième catégorie intermédiaire entre les personnes et les choses, évoquée par les juristes de l'époque comme des choses d'origine humaine, «pour marquer l'origine humaine même dans l'utilisation postérieure, une fois que cela avait été détaché du corps humain». Pour Marie-Angèle Hermitte, "ce souci de marquer la spécificité de l'être humain par rapport

16. Marie-Angèle Hermitte. "Enjeux et stratégies», Droit et génie génétique, Paris, Elsevier, 1994, p. 18.

17. Ibid., p. 19.

Cf. Glowka et al., op. cit., p. 78-79: The FAO Global System for the Conservation and Utilization of Plant Genetic Resources et notamment la définition du droit des agriculteurs : "rights arising from the past, present and future contributions of farmers in conserving, improving and making available plant genetic resources". 
aux autres organismes vivants est l'un des éléments fondamentaux de la construction d'un droit du vivant. II importe, en effet, si l'on ne veut pas tomber dans des confusions qui porteraient atteinte aux droits de l'homme, de marquer juridiquement la différence de l'être humain par rapport aux autres règnes du vivant ${ }^{18}$ ».

Le problème posé est celui du lien social : parler d'une "origine humaine", ici, n'implique pas tant l'existence d'un lien physique que la possibilité de se rapporter à la personne dans la communication et dans le fonctionnement d'une institution sociale. La difficulté que présente la génétique (qu'est-ce qui dans l'être humain mérite un statut différencié: faut-il aller jusqu'aux cellules et aux gènes, au niveau moléculaire, parce qu'ils sont «d'origine humaine»?) n'est pas très différente de l'embarras dans lequel se trouvait à ses débuts il y a deux siècles, ainsi qu'en témoignent des textes de Kant et de Fichte, le régime moderne de la propriété intellectuelle ${ }^{19}$.

Quelle que soit la valeur de cette analogie, d'autres interrogations demeurent au plan juridique, comme l'explique Marie-Angèle Hermitte : "Quel va être le régime juridique marquant la spécificité? On va d'abord dire que l'accès à la ressource humaine implique un consentement de la personne. [...] Mais quelle doit être la précision de ce consentement : doitil porter sur le seul prélèvement ou aussi sur la destination, l'utilisation qui en sera faite? ${ }^{20}$ ".

Ce sont quelques-unes des questions auxquelles il faudrait pouvoir répondre si le patrimoine génétique devaitêtre considéré

18. Hermitte, loc. cit., p. 19.

19. "La possession d'un livre ouvre vers des usages et des pratiques qui ne se réduisent pas à la simple jouissance de sa réalité physique, ni même à l'instrumentation de celle-ci. Qui plus est, l' "esprit" qui est déposé, en tant qu'esprit non seulement "objectif", "fonctionnel", résidant dans le simple usage de l'objet concerné, mais thématisé, communiqué et véhiculé comme tel par lui - ce qui constitue sa spécificité d'objet - renvoie à la difficulté de sa "source", perçue ou non, assumée ou non dans le processus même de la communication" (Jocelyn Benoist, Introduction à Emmanuel Kant, Qu'est-ce qu'un livre? (Textes de Kant et de Fichte), Paris, Presses Universitaires de France, 1995, p. 27).

20. Hermitte, loc. cit., p. 20. 
comme pouvant entrer dans la sphère de l'économie marchande. La logique économique, là où elle est dominante, n'est pas forcément celle du respect de la liberté, de la dignité et de la sécurité des personnes. L'utilitarisme qui en découle ne définit pas ce qu'il faut faire à partir de ce qui est bon, mais à partir de ce qui est profitable. En ce sens, il n'a d'égard ultimement pour aucune forme de solidarité; c'est l'utilité qui compte (et l'efficacité). Contre l'utilitarisme, il faudra toujours être capable de faire appel à ce pouvoir particulier que possède la pensée d'opposer à ce qui "est» (l'ordre des faits), ce qui «doit être» (l'exigence normative). II ne sera peut-être pas superflu de rappeler, alors, l'impératif kantien : «Agis de telle sorte que tu traites l'humanité aussi bien dans ta personne que dans la personne de tout autre toujours en même temps comme une fin, et jamais simplement comme un moyen.»

Le patrimoine génétique humain doit-il, oui ou non, être considéré comme une ressource au même titre que le génome des plantes? II n'y a pas de solution simple: l'application de la Convention de Rio au domaine de la recherche sur la diversité génétique humaine, si elle peut constituer a priori une solution techniquement satisfaisante, pourrait s'avérer un désastre tant du point de vue de l'éthique que du point de vue de l'analyse politique. Si le génome humain devaitêtre considéré simplement comme une ressource, toute démarche d'acquisition des connaissances sur l'hérédité, la génétique des populations et ses prolongements en santé publique risquerait à tout le moins de subir l'effet déplorable des lois du marché. Ce serait le triomphe de la science utilitaire.

Pierre Gendron

Centre de recherche en droit public Université de Montréal 